\title{
Enigma of the second harmonic generation in oriented film of symmetric squaraine
}

Grytsenko, K., Slominski, Y., Tolmachev, O., Resel, R., Ksensov, S., et al.

K. Grytsenko, Y. Slominski, O. Tolmachev, R. Resel, S. Ksensov, S. Schrader, "Enigma of the second harmonic generation in oriented film of symmetric squaraine," Proc. SPIE 6999, Organic Optoelectronics and Photonics III, 699920 (16 April 2008); doi: 10.1117/12.780711

SPIE. Event: SPIE Photonics Europe, 2008, Strasbourg, France 


\title{
Enigma of the second harmonic generation in oriented film of symmetric squaraine
}

\author{
K. Grytsenko ${ }^{1}$ Y. Slominski ${ }^{2}$, \\ O. Tolmachev ${ }^{2}$, R.Resel $^{3}$, S. Ksensov ${ }^{4}$, S. Schrader ${ }^{4}$ \\ ${ }^{1}$ Institute of Semiconductor Physics, 41 Nauki pr., 03028, Kyiv, Ukraine \\ ${ }^{2}$ Institute of Organic Chemistry, 5 Murmanska str., Kyiv, Ukraine \\ ${ }^{3}$ Graz University of Technology, Petersgasse, 16 A-8010 Graz, Austria \\ ${ }^{4}$ Institute of Photonics, Laser \& Plasma Technology, University of Applied Sciences Wildau, \\ Bahnhofstrasse, 15745, Wildau, Germany
}

\begin{abstract}
Second harmonic generation (SHG) in the oriented film of symmetric squaraine (Sq) was studied. Oriented Sq film on aligned polytetrafluoroethylyne (PTFE) sublayer prepared by vacuum deposition with subsequent rubbing using a cloth has been obtained. However, the mechanisms of orientation and SHG are still not clear. Methyl and ethyl substituted hydroxyl-Sq (OHSq) compounds formed oriented films with dichroic ratio of 8 on PTFE layer but with dichroic ratio of 1,5 on Teflon AF. Second layer deposition of Me-OHSq on Et-OHSq or of Et-OHSq on Me-OHSq led to an increase of the film dichroic ratio. Only the film, where Me-OHSq was first layer, exhibits an increase of SHG signal after deposition of second layer. Small differences in bi-layered OHSq films structure was detected by X-Ray diffraction (XRD) spectra.
\end{abstract}

\section{KEYWORDS}

Dye, solid, film, squaraine, optical spectra, deposition, second harmonic generation.

\section{INTRODUCTION}

Since discovery of the second harmonic generation (SHG) in the film of symmetric hydroxy-squaraine (Sq) in 1995 by J. Ashwell et al. ${ }^{1}$ a lot of efforts were made do establish the mechanism of this phenomenon. The opinion about surface induced SHG was spoken by Lynch ${ }^{2,3}$, but Ashwell supported the opinion about aggregate-induced SHG $^{4-8}$. The first results were obtained using Lanmuir-Blodgett deposition method. Later Grytsenko found out, that vacuum deposited and rubbed with a cloth PTFE film can serve as substrate for preparation of oriented organic films. Some squaraines formed oriented films as well ${ }^{9-11}$. But only Me-OHSq film exhibits SHG ${ }^{12,13}$. The difficult task to find the mechanism is due to crystal cell of Me-OHSq is unknown. It was proposed three mechanisms for oriented growth of organic films: 1geometrical matching, 2- topographic adaptation, 3- molecular interactions ${ }^{14}$. Arguments concerned influence of each mechanism were discussed. Our last experimental results seem to support the latter one. But all peculiarities of the influence of the aligned PTFE surface on the oriented growth of organic film were not explained, so further research should be carried out.

The aim of this research is to find more data concerned Squaraine film growth and SHG mechanisms.

*Corresponding author: Tel. +380 445255937 /Fax +380 445255530 ;

e-mail: d.grytsenko@gmail.com; $\underline{\mathrm{d} \_ \text {gryts@isp.kiev.ua }}$ 


\section{EXPERIMANTAL}

Glass plates were covered with PTFE or Teflon AF aligned layer by thermal evaporation with the activation of PTFE vapors by accelerated electrons and rubbing with a cloth (EER) method as described elsewhere ${ }^{10}$. Dyes were synthesized according to the procedures described in detail elsewhere ${ }^{15}$. Dye structure is presented in Fig.1. Dye films were deposited by thermal evaporation in vacuum using a quartz crucible. Pressure in the chamber was maintained at $10^{-3} \mathrm{~Pa}$. Film deposition rate was controlled by quartz crystal microbalance Sigma computer card. Growth rate was in the range of 0.1$1 \mathrm{~nm} / \mathrm{s}$.

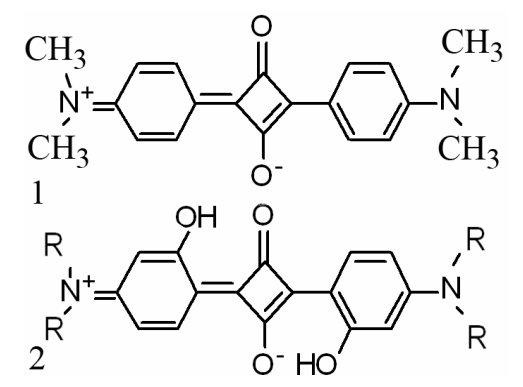

Fig.1. Molecular structure of: 1)- Sq and 2)- OHSq: $\mathrm{R}=$ methyl or ethyl.

Absorption of the films was measured using light, polarised parallel or perpendicular to the rubbing direction. The film surface was studied by AFM NanoScope IIIa Dimension $3000^{\mathrm{TM}}$ microscope. The X-ray diffraction of the films was measured with $\mathrm{CuK \alpha}$ radiation (wavelength 1.54178A) in Bragg-Brentano geometry using a secondary graphite monochromator. Equipment was a SIEMENS D501 powder diffractometer operated in $\Theta / 2 \Theta$ mode. SHG was obtained using picosecond Nd:YAG laser (30 ps, $1064 \mathrm{~nm}, 4 \mathrm{~mJ}$ ). The SHG signal was measured versus sample azimuth angle for pp-, ps-, sp- and ss- polariser/analyser configurations. The intermolecular charge analysis between two molecules in dimers made by B3LYP/6-31G(d,p) quantum chemistry calculations.

\section{RESULTS AND DISCUSSION}

It was found that electronic absorption spectra of SQ films deposited on the aligned PTFE surface show a noticeable dichroism. Sq, methyl and ethyl substituted OHSq compounds formed oriented films with dichroic ratio of 8 on PTFE layer, but with dichroic ratio of 1,5 on Teflon AF. Polarized optical absorption spectra are presented in Fig.2. Longer hydrocarbon tails, i.e. butyl groups, resulted in suppression of any dichroism in absorption spectra. Deposition on substrate kept at $70^{\circ} \mathrm{C}$ led to dichroism increase in Me-OHSq, but decrease in Et-OHSq.

Absorption of Sq and OHSq films showed a broadened spectrum as compared to the monomer spectrum of their solutions. The OHSq film spectrum can be decomposed into three band components, indicating the presence of at least two polymorphs in the film with a strong intermolecular interaction of chromophore groups inside each polymorph ${ }^{11}$. There are two different polymorphs formed by H-aggregation of the OHSq molecules in the form of a "cardpack" arrangement, and J-aggregation in the form of a "slipped stack" arrangement with an overlap of the neighboring donor and acceptor group. The first polymorph shows a sharp absorption maximum at ca. 520-530 nm, whereas the second polymorph results in absorption near $780 \mathrm{~nm}$. According to absorption spectra in Fig.2,2, both polymorphs are present in the films, however, their ratio is different and dependent on the film thickness. In films of small thickness, absorption at $535 \mathrm{~nm}$ prevails, indicating formation of $\mathrm{H}$-aggregates. The second component in spectra of thin films shows absorption at ca. $\sim 640 \mathrm{~nm}$ which matches well with the monomer absorption band in a diluted solution, so presence of monomers in 
thin Sq films can be suggested. In films of a higher thickness the band of J-aggregates at $780 \mathrm{~nm}$ appears, and the band of H-aggregates becomes red-shifted to $\sim 580 \mathrm{~nm}$, with appearance of a strong band at $\sim 690 \mathrm{~nm}$.
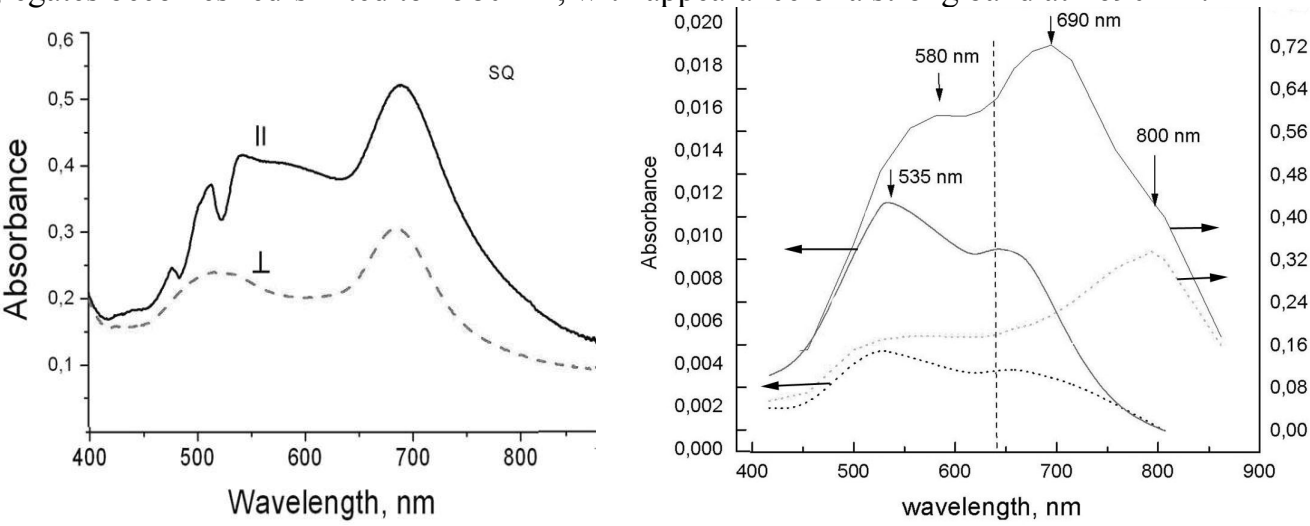

1

2

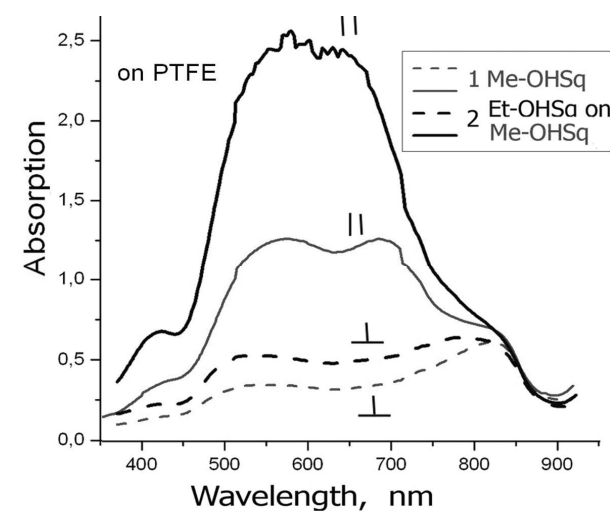

3

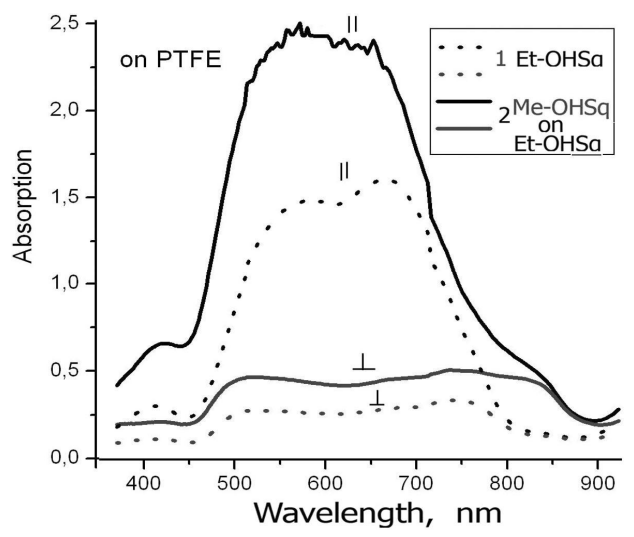

4

Fig.2. Optical absorption spectra of squaraine solid films:

1 - Sq, 2 - OHSq, 3 - Et-OHSq onto Me-OHSq, 4 - Me-OHSq onto Et-OHSq.

The latter two bands are situated symmetrically in respect to the monomer absorption maximum, and their origin cannot be assigned neither to H- or J-aggregates, therefore these should represent some kind of intermediate structure, for example, inclined stacks or a herringbone structure ${ }^{16}$. With increasing film thickness an increase in the dichroic ratio was evident. Absorption profiles of thin films for both the polarization of light are qualitatively the same, and the dichroic ratio is slightly dependent on the wavelength. On the other hand, thick films showed different absorption profiles for different polarization of light in respect to the rubbing direction of the substrate, and their dichroic ratio showed a prominent maximum around $640 \mathrm{~nm}$, while this ratio rapidly decreases to unity as wavelength approaches to $780 \mathrm{~nm}$. This behaviour points out that J-aggregates do not significantly contribute to the increase of the dichroic ratio of films grown on the aligned PTFE substrates.

OHSq clusters did not show visible orientation in AFM images (Fig.3) independent on the preparation technique of the PTFE layer ${ }^{11}$. Cluster size increases with film thickness growth together with dichroic ratio. 

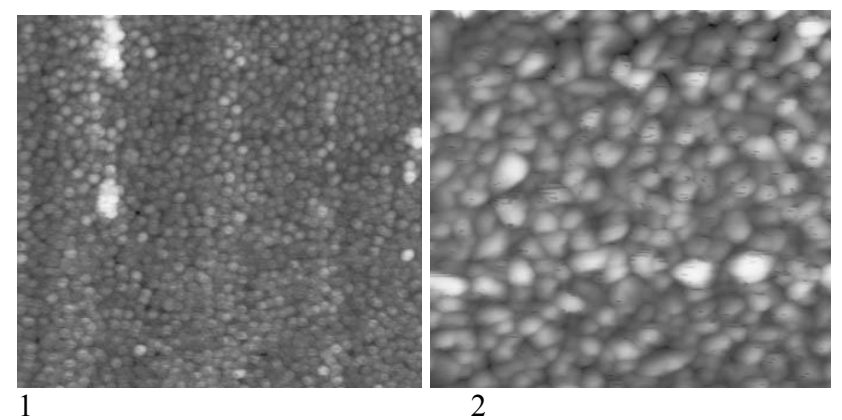

Fig.3. AFM images of the thin and thick OHSq films: $1-5 \mathrm{~nm}$ thick, $2-30 \mathrm{~nm}$ thick. Size: 1 x $1 \mu \mathrm{m}$.

Second layer deposition of Me-OHSq on Et-OHSq or of Et-OHSq on Me-OHSq led to an increase of the both bilayered films dichroic ratio (Fig.2,3 and 2,4). Only small differences in the film structure were found out by XRD (Fig.4). The small peak at $d=4.95 \AA$ appears in all samples with comparable intensity, it represents the characteristic feature of the PTFE thin film (according to the database PDF no.47-2217).
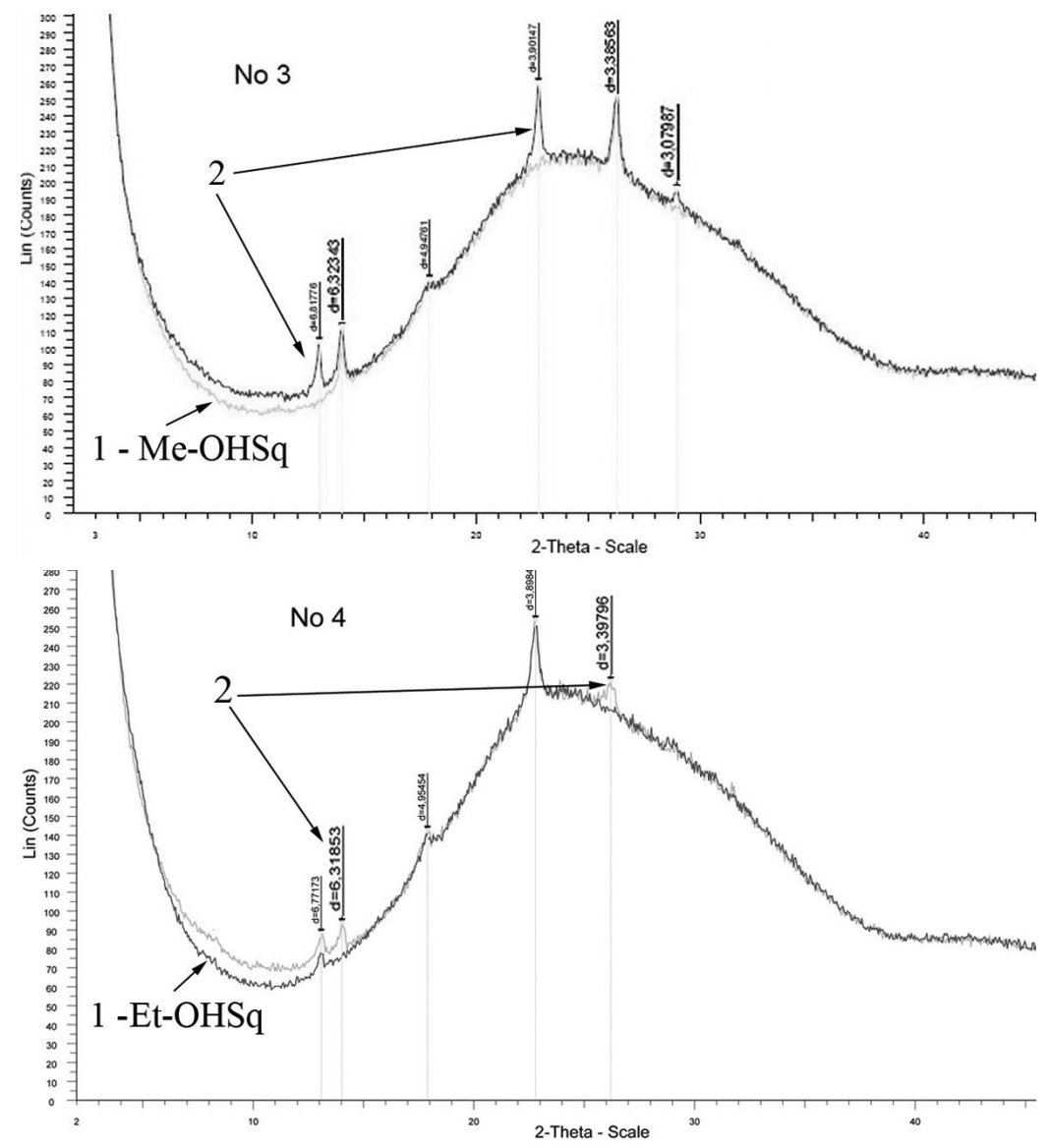

Fig.4. XRD spectra of one and bilayered OHSq films: sample No 3: 1 - Me-OHSq onto PTFE, 2 - Et-OHSq onto Me-OHSq, sample No 4) 1 - Et-OHSq onto PTFE, 2 - Me-OHSq onto Et-OHSq. 
XRD peaks of the Me-OHSq are visible at 6.32A and 3.38A, in case of the bilayer the peaks of Et-OHSq at $6.82 \mathrm{~A}, 3.90$ $\mathrm{A}$ and $3.08 \mathrm{~A}$ arise (No 3). In No 4 the peaks of Et-OHSq and Me-OHSq are visible. Remarkable is that the peak at $3.9 \mathrm{~A}$ in sample 4 is more pronounced in comparison to the other peaks and to sample 3 . This indicates a slightly different preferred orientation of the crystallites within the film. This preferred orientation is visible for Et-OHSq as well as for next layer of Me-OHSq. The heteroepitaxy of OHSq molecules onto rubbed PTFE surface and heteroepitaxy of MeOHSq onto Et-OHSq and vice versa can be suggested, but need farther confirmation. But final crystal structure formed in bilayered films is slightly different and dependent on the structure of the first layer.

Sample 3 showed SHG increase after deposition of the second layer (Fig.5), while sample 4 showed no SHG at all. Two peaks are more intensive in sample No 3: 6,32 and 3,38 Angstroms. Moreover, namely these peaks have increased intensity with Et-OHSq deposition on Me-OHSq film. The film with crystal structure produced these peaks, is responsible for SHG.

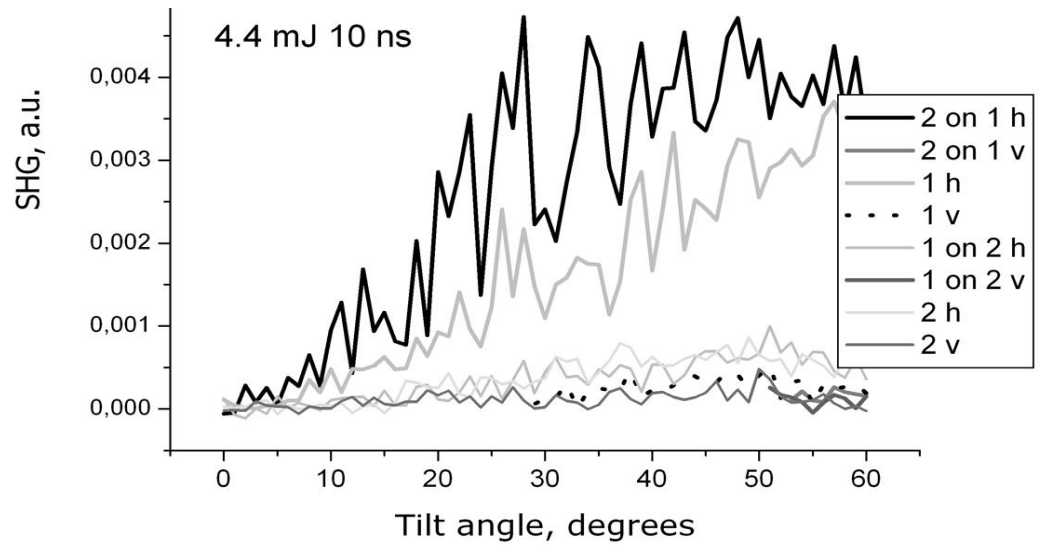

Fig.5. SHG spectra of OHSq one- and bilayered films: Sample No3) 1 - Me-OHSq onto PTFE, 2 - Et-OHSq onto MeOHSq, Sample No4) 2 - Et-OHSq onto PTFE, 1 - Me-OHSq onto Et-OHSq.

The polarization-depended signal of SHG from Me-OHSq were observed (Fig. 6).

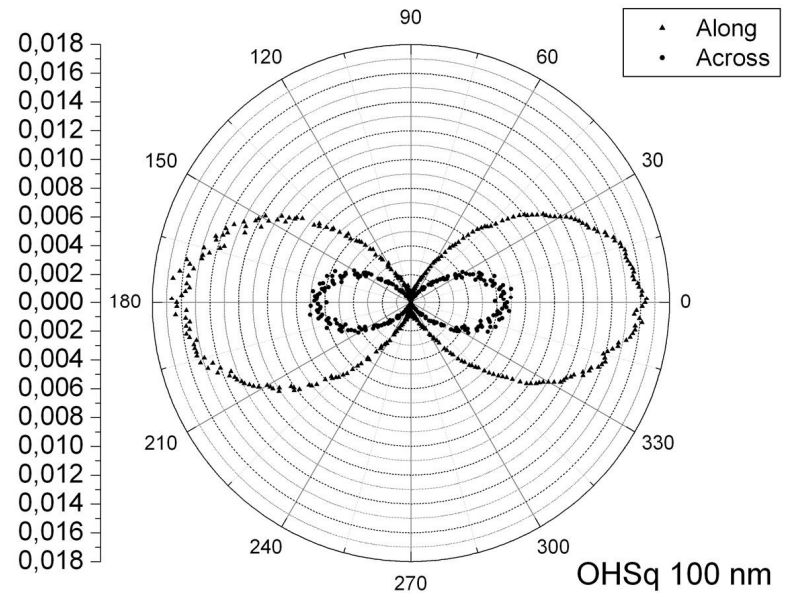

Fig.6. The SHG intensity of a 100nm Me-OHSq layer versus polarization angle (zero and 90 degrees correspond s and $\mathbf{p}$ polarization respectively). The angle between the substrate and orienting frictions directions is denoted as across (orthogonal) and along (collinear). 


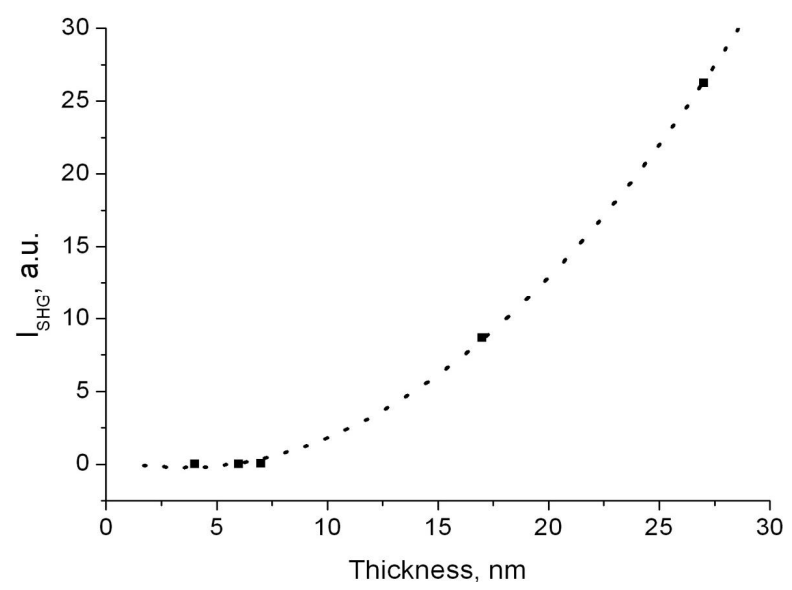

Fig.7. Thickness depended SHG from Me-OHSq film.

Centrosymmetrical molecule can posses SHG on interface, where centrosymmetry is broken. The vacuum-deposited layer of Me-OHSq demonstrates increase of SHG with film thickness growth (Fig.7). This is the evidence of volume nature of SHG in the OHSq film.

The internal molecular structure and electronic density distribution of single molecule of Sq and Me-OHSq were investigated theoretically. Both semi-empirical (PM3) and ab-initio (HF/6-311G) methods showed that both of single molecules are centrosymmetrical. This means, that the molecule itself cannot generate a second harmonic (excluding interface monolayer). Furthermore, calculated dipole momentum of both of molecules have values less than 0.001 Debye. The probable source of nonlinear properties is the intermolecular interactions. As know from works of Ashwell, the squaraine molecules are able to form aggregates with high second-order nonlinear activity ${ }^{6-8}$. XRD structural analysis of the SHG-active 2,4-bis(4-(N,N-dibutylamino)phenyl)squaraine made by Ashwell et. al. ${ }^{8}$ has shown the chromophore is linear and planar and has an inversion center. Intermolecular charge transfer between the terminal donor and central acceptor of adjacent molecules can give rise to acentric "T-shaped" dimers and higher aggregates. These are supposed as the "molecular" building blocks for SHG. Since the formation of second order active aggregates involves the central acceptor part of molecule (-H and -OH groups) together with donor end groups (methyl, ethyl) the arranging influence of substrate plays role by forming the initial microcrystalline layer.

To analyse charge exchange between molecules, the quantum chemical molecular optimization were performed. We selected B3LYP method of differential functional theory (DFT) with 6-31G(d,p) basis (Table). All dimers have non zero dipole momentum, that point to possibility of dipole-dipole interaction between aggregates. We also observed highest charge exchange in case of SHG-active Me-OHSq molecular dimer.

Table

\begin{tabular}{|l|l|l|}
\hline Molecule & Dipole, $D$ & $\begin{array}{l}\text { Intermolecular charge } \\
\text { transfer, } e\end{array}$ \\
\hline $2 \mathrm{Me}-\mathrm{Sq}$ & 0.1039 & 0.0017 \\
\hline $2 \mathrm{Me}-\mathrm{OHSq}$ & 0.2303 & 0.0472 \\
\hline $2 \mathrm{Eth}-\mathrm{Sq}$ & 0.2392 & 0.0104 \\
\hline 2 Eth-OHSq & 0.1818 & 0.0004 \\
\hline
\end{tabular}

The system of two molecules has nonzero dipole moment and non-symmetrical charge distribution. So single dimer can generate second harmonic, but quadratic SHG increase with film thickness growth shows, that main part in SHG is from crystal cell, which should be non symmetrical. 


\section{CONSLUSIONS}

It was found that oriented growth of squaraine films on the aligned Teflon surface is strongly affected by the molecular structure of the dye and kind of Teflon used. Only PTFE sublayer led to formation of dye film with high dichroic ratio. Formation of dye aggregates affects film optical properties, but nor H-aggreagtes, neither $\mathrm{J}$ ones are responsible for dicroism. Perhaps, all three mechanisms: geometrical matching, topographic adaptation and molecular interactions are taking place in formation of oriented film with different contributions in dependence on molecular properties of the growing film, sublayer properties and deposition conditions. We suggested that heteroepitaxy occurs when OHSq derivatives are grown on PTFE and when they are grown one on the other.

Second harmonic generation in the films, composed from symmetric dye molecules, is still not explained fenomenon. We just showed, that it is originated from film bulk, but not from surface.

\section{ACKNOWLEDGMENTS}

Financial support with STCU Grant 3480 AND NATO CL Grant is gratefully acknowledged.

\section{REFERENCES}

[1] G.J. Ashwell, G. Jefferies, D.G. Hamilton, et al., "Strong second-harmonic generation from centrosymmetric dyes", Nature, 375, 385-388, 1995.

[2] D.E. Lynch,. U. Geissler, R. I. Peterson, et al. "Synthesis and Solid-State Structure of the Unsymmetrical Squaraine Dye", J. Chem. Soc., Perkin Trans. 2, 827, 1997.

[3] D.E. Lynch, I.R. Peterson, M.Floersheimer, et al., "Synthesis and non-linear optical properties of ( $N$-alkylpyrrol-2yl)-squaraine derivatives”, Part 2, J. Chem. Soc., Perkin Trans. 2, 779 (1998).

[4] G.J. Ashwell, G. Jefferies, N. D. Rees, et al., "Centrosymmetric Chromophores: Second-Harmonic Generation from Langmuir-Blodgett and Spun-Coated Films of Hydroxy-Substituted Squaraines", Langmuir, 14, 2850-2856 (1999).

[5] G.J.Ashwell, A.N.Dyer and A.Green, "Diminished Second-Harmonic Generation from Oligomeric Squaraine Derivatives of $\mathrm{N}$-Octadecylpyrrole Relative to the Intensity from Films of the Centric Anilinosquaraines", Langmuir 15, 3627-3631, (1999).

[6] G. J. Ashwell, "Centrosymmetric molecules for second harmonic generation”, Adv. Materials, 8, 248-250 (1996).

[7] G.J.Ashwell, T.Handa, P.Leeson, K.Skjonnemand, G. Jefferies, and A. Green, "Second-harmonic generation from Langmuir-Blodgett films of centrosymmetric squaraines with heterocyclic donor groups", J. Materials Chem., 8, 377382 (1998).

[8] G.J.Ashwell, G.S.Bahra, C.R.Brown, D.G.Hamilton, C. H. L. Kennard, and D. E. Lynch, "2,4-Bis[4-(N,Ndibutylamino)phenyl]squaraine: X-ray crystal structure of a centrosymmetric dye and the second-order non-linear optical properties of its non-centrosymmetric Langmuir-Blodgett films”, J. Materials Chem., 6, 23-26 (1996).

[9] Gritsenko, K.P., Slominski, Yu.L., Tolmachev, A.I., Tanaka, T., Schrader, S., Brehmer, L., Thierry, A., Wittmann, J.C., "Anisotropy of dye films deposited onto oriented PTFE sublayers", Proc. SPIE, 4833, 482-486 (2003).

[10] K.P. Gritsenko, A.M. Krasovsky, "Thin film deposition of polymers by vacuum degradation", Chemical Reviews, 103, No 9, 3607-3650 (2003).

[11] K.P.Gritsenko, D.O.Grinko, O.P.Dimitriev, S.Schrader, A.Thierry, J.C.Wittmann, "Oriented Growth of Squaraine Dyes on Uniaxially Aligned Poly(tetrafluoroethylene) Surfaces", Optical Memory and Neural Networks, N3, pp.135143, 2004.

[12] V,Ksensov, K.Grytsenko, A.Thierry, A.I.Tolmachev, S.Schrader, "Strong second-order nonlinear responce from vacuum-deposited thin film of centrosymmetrical OHSQ dye", X International Conference on Quantum Optics'2004 (ICQO'2004), May 30- June 3, 11 (2004). 
[13] K.Grytsenko, V.Bogutskii, Yu.L.Slominskii, O.I.Tolmachev, R.Resel, S.Ksensov, S.Schrader, "Heteroepitaxial growth of Squaraine bilayered films onto rubbed polytetrafluoroethylene surface", Abstr. Book: Conf. ECOER07, Varenna, September 30 - October 4, 43 ( 2007).

[14] A. Thierry, J.C. Wittmann, B. Lotz, V. da Costa, et al., "Organisation, structure and morphology of organic thin films via electron microscopy“", Org. Electronics, 5, 7-22 (2004).

[15] A.I. Tolmachev, A.Ya. Il'chenko, "Polymethine Dyes“, J. Sci. Appl. Photo. 42, 483 (2001).

[16] M.E Stawasz, D.L. Sampson, B.A. Parkinson, "Scanning Tunneling Microscopy Investigation of the Ordered Structures of Dialkylamino Hydroxylated Squaraines Adsorbed on Highly Oriented Pyrolytic Graphite“, Langmuir, 16, 2326 (2000). 